\begin{tabular}{|c|l|}
\hline Title & $\begin{array}{l}\text { Mitochondrial delivery of mastoparan with transferrin liposomes equipped with a pH- sensitive fusogenic peptide for } \\
\text { selective cancer therapy. }\end{array}$ \\
\hline Author(s) & $\begin{array}{l}\text { Yamada, Y uma; Shinohara, Y asuo; Kakudo, Tomoyuki; Chaki, Shinji; Futaki, Shiroh; Kamiya, Hiroy uki; Harashima, } \\
\text { Hidey oshi }\end{array}$ \\
\hline Citation & $\begin{array}{l}\text { International journal of pharmaceutics, 303(1-2), 1-7 } \\
\text { https://doi.org/10.1016/.ijpharm.2005.06.009 }\end{array}$ \\
\hline Issue Date & 2005-10-13 \\
\hline Doc URL & http://hdl.handle.net/2115/48155 \\
\hline Type & article \\
\hline File Information & 1 IntJPharm.pdf \\
\hline
\end{tabular}

Instructions for use 


\section{Mitochondrial delivery of mastoparan with transferrin liposomes equipped with a pH-sensitive fusogenic peptide for selective cancer therapy}

Yuma Yamada $^{1}$, Yasuo Shinohara ${ }^{2}$, Tomoyuki Kakudo ${ }^{1}$, Shinji Chaki ${ }^{1}$, Siroh Futaki ${ }^{3}$, Hiroyuki Kamiya $^{1}$ and Hideyoshi Harashima ${ }^{1,4}$

${ }^{1}$ Graduate School of Pharmaceutical Sciences, Hokkaido University, Kita-12, Nishi-6, Kita-ku, Sapporo 060-0812, Japan

${ }^{2}$ Division of Gene Expression, Institute for Genome Research, The University of Tokushima, Kuramoto-cho 3-18, Tokushima 770-8503, Japan

${ }^{3}$ Institute for Chemical Research, Kyoto University, Uji, Kyoto 611-0011, Japan.

${ }^{4}$ Corresponding author:

Hideyoshi Harashima, Ph. D.

Laboratory for Mocelular Design of Pharmaceutics

Graduate School of Pharmaceutical Sciences, Hokkaido University

Kita 12, Nishi 6, Kita-ku, Sapporo 060-0812, Japan

Tel\&FAX +81-11-706-3704

E-mail harasima@pharm.hokudai.ac.jp

Keywords: mastoparan, transferrrin liposomes, GALA, mitochondrial delivery, anticancer therapy, apoptosis. 


\begin{abstract}
Mastoparan (MP), a potent facilitator of mitochondrial permeability transition (PT), could be used as an antitumor agent, if it were encapsulated in a tumor selective delivery system. We recently developed transferrin-modified liposomes (Tf-L) with a $\mathrm{pH}$-sensitive fusogenic peptide (GALA), which delivers an encapsulated fluorescent marker into cytosol efficiently. In this study, we encapsulated MP into Tf-L with GALA for the selective delivery to mitochondria of tumor cells. The MP showed potent PT activity at concentrations above $25 \mu \mathrm{M}$ in a homogenate of $\mathrm{K} 562$ cells as well as in isolated mitochondria in the presence of phosphate. Tf-L equipped with cholesteryl GALA can release encapsulated sulforhodamine B, while Tf-L failed, as evidenced by confocal laser scanning microscopy. The MP, which was delivered with Tf-L with GALA, released cytochrome c (cyt c) from mitochondria to the cytosol, while free MP released cyt $\mathrm{c}$ not only to the cytosol but also extracellulary. These results demonstrate the utility of MP in Tf-L with GALA for cancer therapy.
\end{abstract}




\section{Introduction}

Mastoparan (MP) is a 14-amino acid amphipathic peptide obtained from wasp venom (Zimmerberg et al., 1986) and it has been reported to induce a potent mitochondrial permeability transition (PT) in the range of concentration between 5 $100 \mu \mathrm{M}$ by forming a permeability transition pore (PTP) (Pfeiffer et al., 1995). Therefore, MP could be used as an antitumor agent, if it could be delivered to tumor cells selectively, otherwise it would kill normal cells because of its potent PT activity.

We recently developed transferrin-modified liposomes (Tf-L) to target tumor cells which up-regulate Tf receptors. Tf-L can be internalized by tumor cells via Tf receptor-mediated endocytosis, but the encapsulated fluorescent marker is trapped in endosomal/ lysosomal compartments and is not recycled to the plasma membrane. To solve this problem, we introduced a $\mathrm{pH}$-sensitive fusogenic peptide, GALA, into Tf-L to enhance cytosolic release. We found that the GALA peptide, which was introduced into liposomal membrane as cholesteryl GALA (Chol-GALA), not in an aqueous phase as a free form, was able to exert cytosolic release activity (Kakudo et al., 2004).

In this study, we encapsulated MP into Tf-L equipped with Chol-GALA to release cytochrome $\mathrm{c}(\mathrm{cyt} \mathrm{c})$ selectively in tumor. MP in free form can release cyt $\mathrm{c}$ not only in the cytosol but also to the medium, which indicates the non-specific action of this peptide. While, the Tf-L equipped with Chol-GALA has the ability to deliver MP to target tumor cells and it is internalized via a $\mathrm{Tf}$ receptor-mediated endocytosis and successfully delivers the MP to the cytosol without producing a non-specific action. 


\section{Experimental}

\subsection{Materials}

K562 cells, human chronic myelogenous leukemia cells were obtained from the National Institute of Health Sciences (Japan). Roswell Park Memorial Institute (RPMI) 1640 medium, fetal bovine serum (FBS) were purchased from Invitrogen (Carlsbad, CA). A specific antibody against cyt c was prepared as described previously (Shinohara et al., 2002). The peptide chain of the MP (Pfeiffer et al., 1995) was synthesized using a Shimadzu automated solid-phase peptide synthesizer, model PSSM-8. Egg yolk phosphatidylcholine (EPC), cholesterol (Chol) and N-[3-(2-pyridyldithio)propionate]-1,2-dipalmitoyl-sn-glycero-3-phosphatidylethanolami ne (PDP-PE) were purchased from Avanti Polar lipids (Alabaster, AL). Tf was obtained from Sigma (St. Louis, MO). Sulforhodamine B (Rho) was purchased from Molecular Probes (Eugene, OR). Chol-GALA was synthesized using a previously described procedure (Futaki et al., 1997; Kakudo et al., 2004).

\subsection{Cell culture and preparation of homogenate solution, mitochondrial fraction}

K562 cells were cultured in RPMI 1640 medium supplemented with 10\% FBS under an atmosphere of $5 \% \mathrm{CO}_{2} /$ air at $37^{\circ} \mathrm{C}$.

A homogenate solution and a mitochondrial fraction were obtained from a total of $3.0 \times 10^{7}$ cultured K562 cells. Unless stated otherwise, all operations were performed on ice. The cells were washed with ice-cold phosphate-buffered saline (PBS $(-))$, resuspended in $1 \mathrm{~mL}$ of mitochondria isolation buffer (MIB) consisting of $0.25 \mathrm{M}$ sucrose, $1 \mathrm{mM}$ EDTA, $2 \mathrm{mM}$ Tris-HCl (pH7.4), and subjected to 20 strokes in a Dounce homogenizer to give a homogenate. The mitochondrial fraction was obtained from the homogenate solution using a differential centrifugation technique. The homogenate solution was centrifuged at $700 \mathrm{x} g$ for $10 \mathrm{~min}$. The supernatant was then centrifuged at 
$16,000 \mathrm{x} g$ for $10 \mathrm{~min}$ to pellet the mitochondria. The pelleted mitochondrial fraction was resuspended in $50 \mu \mathrm{L}$ MIB. This mitochondrial fraction was stored on ice prior to use.

\subsection{Release activity of cyt c from mitochondria}

A $5 \mu \mathrm{L}$ aliquot of the MP was added to $50 \mu \mathrm{L}$ of mitochondrial fraction in the presence and absence of $10 \mathrm{mM}$ phosphate and incubated at $25^{\circ} \mathrm{C}$ for $10 \mathrm{~min}$. The suspension was then centrifuged at $20,400 \times \mathrm{g}$ for $10 \mathrm{~min}$ at $4{ }^{\circ} \mathrm{C}$, and the supernatant retained as the supernatant fraction $(\mathrm{S})$. The pelleted mitochondrial fraction was resuspended in $50 \mu \mathrm{L}$ of PBS (-) to give the pellet fraction (P).

A $60 \mu \mathrm{L}$ aliquot of the MP was added to $540 \mu \mathrm{L}$ of homogenate in the presence and absence of $10 \mathrm{mM}$ phosphate and incubated at $25^{\circ} \mathrm{C}$ for $10 \mathrm{~min}$. The suspension was then centrifuged at $700 \times g$ for $10 \mathrm{~min}$ on $4^{\circ} \mathrm{C}$. The supernatant was centrifuged at $16,000 \times \mathrm{g}$ for $10 \mathrm{~min}$, the resulting supernatant was retained as the supernatant fraction (S). The pelleted mitochondrial fraction was resuspended in $100 \mu \mathrm{L}$ of PBS (-) to give the pellet fraction (P).

\subsection{Western blotting}

$10 \mu \mathrm{L}$ of sample was mixed with $10 \mu \mathrm{L}$ of SDS-gel-loading buffer $(100 \mathrm{mM}$ Tris- $\mathrm{HCl} \quad(\mathrm{pH} \quad 6.8), \quad 4 \% \quad$ SDS, 12\% 2 -mercaptoethanol, $20 \%$ glycerol, $\quad 0.05 \%$ bromophenol blue), subjected to $15 \%$ SDS-PAGE, and then electroblotted onto an Nitrocellulose filter (BM Equipment, Japan). Rabbit anti cyt c antibodies were used at a 1:1000 dilution and secondary HRP-conjugated anti rabbit antibodies (Amersham Biosciences, UK) were used at a 1:1000 dilution. The intensities of the immunodetected protein band corresponding to cyt $\mathrm{c}$ were determined by using the NIH image v.1.63 software. 


\subsection{Preparation of Tf-modified liposomes equipped with Chol-GALA}

Liposomes were composed of EPC and Chol (2:1 molar ratio), and for cross-linking with Tf, PDP-PE was incorporated at $1.0 \mathrm{~mol} \%$ of the total lipid. Liposomes were prepared by reverse phase evaporation (Suzoka et al., 1978) followed by extrusion with a Mini Extruder (Avanti Polar Lipids), through 200 and $100 \mathrm{~nm}$ polycarbonate filters (Nuclepore), 11 times for each pore size. Rho was used as an aqueous phase marker. Unencapsulated Rho was separated by size-exclusion chromatography on a Bio-gel A 1.5 m Column (100-200 mesh, BIO RAD) equilibrated with PBS (-). MP was encapsulated as the aqueous phase using a similar procedure. Chol-GALA was introduced into the liposomal membrane at 2 mol \% of the total lipids. In this study, the encapsulation efficiencies of MP was assumed to be same as that of rhodamine which is frequently used as an aqueous marker of liposomes.

The attachment of Tf to liposomes was accomplished as described previously (Kakudo et al., 2004). The particle sizes of the liposomes were measured by means of dynamic light scattering spectrophotometry (ELS-8000, Photal Otsuka Electronics).

\subsection{Intracellular trafficking of liposomes}

K562 cells $\left(5 \times 10^{4}\right.$ cells $\left./ \mathrm{mL}\right)$ in serum free medium were incubated with liposomes in which Rho was encapsulated as an aqueous phase maker (final lipid concentration of $630 \mu \mathrm{M}$ ) for $6 \mathrm{hr}$ in the presence of $\mathrm{Fe}_{2}\left(\mathrm{SO}_{4}\right)_{3}$-EDTA (final $\mathrm{Fe}^{3+}$ concentration of $100 \mu \mathrm{M})$. After incubation, the cells were washed twice with ice-cold PBS (-) and analyzed by confocal laser microscopy.

2.7. Release activity of cyt c from mitochondria in living cells by Tf-L equipped with Chol-GALA encapsulating MP 
A $30 \mu \mathrm{L}$ aliquot of MP, Tf-L equipped with Chol-GALA encapsulating MP, and MIB were individually added to $300 \mu \mathrm{L}$ of a K562 cell suspension $\left(6 \times 10^{7}\right.$ cells $/ \mathrm{mL}$ $\mathrm{K} 562$ cells in serum free medium), and incubated at $37^{\circ} \mathrm{C}$ for $10 \mathrm{~min}$ (final $\mathrm{MP}$ concentration of $100 \mu \mathrm{M}$ ) in the presence of $\mathrm{Fe}_{2}\left(\mathrm{SO}_{4}\right)_{3}$-EDTA (final $\mathrm{Fe}^{3+}$ concentration of $100 \mu \mathrm{M})$.

The suspension was centrifuged at $110 \times \mathrm{g}$ for $3 \mathrm{~min}$ at $4^{\circ} \mathrm{C}$, the supernatant was retained as the medium fraction $(\mathrm{Me})$. After the removing supernatant, the pellet was washed with ice-cold PBS (-), resuspended in $330 \mu \mathrm{L}$ of MIB, and subjected to 20 strokes in a Dounce homogenizer. The homogenate solution was then centrifuged at 700 $\mathrm{x} g$ for $10 \mathrm{~min}$ at $4^{\circ} \mathrm{C}$. The supernatant was centrifuged at $16,000 \times \mathrm{g}$ for $10 \mathrm{~min}$, the supernatant obtained was retained as the cytosol fraction (Cy). The pelleted mitochondria fraction was resuspended in $50 \mu \mathrm{L}$ of PBS (-) to give the mitochondrial fraction (Mt). Each sample was analyzed by western blotting. 


\section{Results and Discussion}

\subsection{PT induction by MP}

MP is a potent facilitator of PT (Pfeiffer et al., 1995). To determine the concentration of MP necessary for PT, MP was added to mitochondria isolated from K562 cells, and the cell homogenate. Cyt c was detected by western blotting using its specific antibodies. The cyt $\mathrm{c}$ release activity of MP was first estimated in the isolated mitochondria. MP (final concentration of $50 \mu \mathrm{M}$ ) was added to the isolated mitochondria in the presence and absence of phosphate, and then incubated at $25^{\circ} \mathrm{C}$ for 10 min. As previously reported (Pfeiffer et al., 1995), cyt c was released from the mitochondria by the added MP in the presence of phosphate (Fig. 1), although the precise role of phosphate is unknown. We next measured the cyt c release activity of MP in a K562 cell homogenate, which are a better model for living cells than isolated mitochondria. MP (final concentration of $50 \mu \mathrm{M}$ ) was added to the $\mathrm{K} 562$ homogenate in the presence and absence of phosphate, followed by incubation at $25^{\circ} \mathrm{C}$ for $10 \mathrm{~min}$. In contrast to the isolated mitochondria, cyt $\mathrm{c}$ was also released from mitochondria in the absence of phosphate (Fig. 1). The amounts of cyt c released by various concentrations of MP, with or without phosphate, were similar, as estimated by quantitative western blotting (Fig. 2). Approximately $\sim 50 \%$ of the cyt c was released from the mitochondria in both cases upon the addition of $50 \mu \mathrm{M}$ MP. The MP showed potent activity for PT, at concentrations above $25 \mu \mathrm{M}$. These results suggest that the concentration of phosphate in the homogenate was sufficient for the release of cyt c. Thus, the delivery of more than $25 \mu \mathrm{M}$ MP into the cytosol could induce PT and, hence, exert cytotoxic effects.

\subsection{Efficient internalization and endosomal escape by Tf-L equipped with Chol-GALA}

The cyt c release activity of MP makes it as an attractive antitumor agent when a tumor-selective delivery system is used. We recently developed a Tf-L to target 
tumor cells which up-regulate Tf receptors (Kakudo et al., 2004). In addition, we also found that the delivery of the contents into the cytosol by $\mathrm{pH}$-sensitive fusogenic peptide GALA was efficient. Thus, MP in the Tf-L equipped with Chol-GALA effectively exerted cytotoxic activity in tumor cells. To achieve the targeted delivery of MP into tumor cells, we had planned to use this Tf-L equipped with Chol-GALA encapsulating MP. When Rho was encapsulated, Tf-L with GALA effectively delivered the fluorescence marker into the cytosol of K562 cells (Fig. 3). In contrast, Tf-L without GALA did not release the marker into the cytosol, suggesting that it remained trapped in endosomes/lysosomes.

\subsection{Cytotoxic effects of MP encapsulated in liposomes}

To examine the usefulness of encapsulating MP into Tf-L equipped with Chol-GALA, K562 cells were treated with MP in the liposomes and in free form, and the induction of PT was compared.

When MP (final concentration of $100 \mu \mathrm{M}$ ) was directly added to a K562 culture medium, cyt c was detected in the culture medium as well as in the cytosol (Fig. 4). This indicates that MP entered the K562 cells through the cell membrane (Matsuzaki et al., 1996), and cyt c, after release from the mitochondria, leaked out through the disrupted cell membrane. Thus, encapsulation appears to be highly important in preventing the non-specific action of this peptide. On the other hand, Tf-L equipped with Chol-GALA encapsulating MP (final concentration of $100 \mu \mathrm{M}$ as MP) was added to a K562 cell culture medium, cyt c was detected in the cytosol but not in the culture medium (Fig. 4). A calculation indicated that $48 \%$ of the cyt c was released from the mitochondria. Thus, MP was successfully delivered into the cytosol without producing any non-specific action, and the encapsulated MP peptide did not leak from the liposomes to outside the cells. These results provide a clear demonstration of the utility 
of MP in Tf-L equipped with Chol-GALA for cancer therapy.

\subsection{Towards use of MP as an antitumor reagent}

The findings herein show that Tf-L equipped with Chol-GALA encapsulating MP is a potentially useful cytotoxic agent that is selective for tumor cells. These types of liposomes, when their diameters are adjusted to $\sim 100 \mathrm{~nm}$, could deliver drugs to tumor cells overexpressing the Tf-receptor in vivo (Ciechanover et al., 1983; Ishida et al., 2001). This cell-selective incorporation of liposomes would be expected to occur via receptor-mediated endocytosis (Fig. 5). Attaching the $\mathrm{pH}$-sensitive fusogenic peptide GALA to the surface of the liposomes facilitates their endosomal escape (Kakudo et al., 2004). In addition, Tf-L without GALA encapsulating MP did not induce PT in our preliminary experiments (data not shown), indicating importance of the GALA attachment. MP, after escaping from both liposomes and endosomes, can induce the PT of mitochondria, thus releasing cyt $\mathrm{c}$ is released (Fig. 5). This proposed intracellular trafficking of MP could explain the results obtained in this study (Fig. 4).

When Tf-L equipped with Chol-GALA encapsulating MP was added to K562 cells, $48 \%$ of the cyt c was released from the mitochondria. We roughly estimate that the concentration of MP in the cytosol was $\sim 50 \mu \mathrm{M}$ based on the ratio of released cyt c to the total cyt $\mathrm{c}$ (Fig. 2). This suggests that internalization of the liposomes into the cells and the endosomal escape of MP were highly effective. To use the MP as an antitumor agent, further improvements of in delivery system will be necessary. Studies concerning this are currently in progress. 


\section{Acknowledgments}

This work was supported, in part, by Grants-in-Aid from the Ministry of Education, Culture, Sports, Science and Technology of Japan, by the Japan Society for the Promotion of Science and by the Nagase Science and Technology Foundation. 


\section{References}

Ciechanover, A., Schwartz, A. L., Dautry-Varsat, A., and Lodish, H. F., 1983. Kinetics of internalization and recycling of transferrin and the transferrin receptor in a human hepatoma cell line. Effect of lysosomotropic agents. J Biol Chem, 258, 9681-9689.

Futaki, S., Ishikawa, T., Niwa, M., Kitagawa, K., and Yagami, T., 1997. Embodying a stable alpha-helical protein structure through efficient chemical ligation via thioether formation. Bioorg Med Chem, 5, 1883-1891.

Ishida, O., Maruyama, K., Tanahashi, H., Iwatsuru, M., Sasaki, K., Eriguchi, M., and Yanagie, H., 2001. Liposomes bearing polyethyleneglycol-coupled transferrin with intracellular targeting property to the solid tumors in vivo. Pharm Res, 18, 1042-1048.

Kakudo, T., Chaki, S., Futaki, S., Nakase, I., Akaji, K., Kawakami, T., Maruyama, K., Kamiya, H., and Harashima, H., 2004. Transferrin-modified liposomes equipped with a pH-sensitive fusogenic peptide: an artificial viral-like delivery system. Biochemistry, 43, 5618-5628.

Matsuzaki, K., Yoneyama, S., Murase, O., and Miyajima, K., 1996. Transbilayer transport of ions and lipids coupled with mastoparan X translocation. Biochemistry, 35, $8450-8456$.

Pfeiffer, D. R., Gudz, T. I., Novgorodov, S. A., and Erdahl, W. L., 1995. The peptide mastoparan is a potent facilitator of the mitochondrial permeability transition. J Biol Chem, 270, 4923-4932. 
Shinohara, Y., Almofti, M. R., Yamamoto, T., Ishida, T., Kita, F., Kanzaki, H., Ohnishi, M., Yamashita, K., Shimizu, S., and Terada, H., 2002. Permeability transition-independent release of mitochondrial cytochrome $\mathrm{c}$ induced by valinomycin. Eur J Biochem, 269, 5224-5230.

Szoka, F., Jr. and Papahadjopoulos, D., 1978. Procedure for preparation of liposomes with large internal aqueous space and high capture by reverse-phase evaporation. Proc Natl Acad Sci U S A, 75, 4194-4198.

Zimmerberg, J. and Parsegian, V. A., 1986. Polymer inaccessible volume changes during opening and closing of a voltage-dependent ionic channel. Nature, 323, 36-39. 


\section{FIGURE LEGENDS}

Figure 1. PT induction by MP in isolated mitochondria and a K562 cell homogenate. MP (final concentration of $50 \mu \mathrm{M}$ ) was added to the isolated mitochondria and the K562 cell homogenate in the presence and absence of phosphate (Pi), followed by incubation at $25^{\circ} \mathrm{C}$ for $10 \mathrm{~min}$. The pellet fraction $(\mathrm{P})$ and the supernatant fraction (S) were prepared as described in "Materials and Methods". Both fractions were subjected to a western blotting analysis using a specific antibody against cyt c. Lane 1, rat liver mitochondria (RLM); lanes 2-5, isolated mitochondria; lanes 6-9, homogenate of K562 cells. Please note that the Mt fraction was six times more concentrated than the Cy fraction in the experiments using the K562 cell homogenate.

Figure 2. Amount of cyt c released from mitochondria by various concentrations of MP. MP (final concentrations of $25,50,75,100 \mu \mathrm{M}$ ) was added to the $\mathrm{K} 562$ cell homogenate in the presence and absence of phosphate, followed by incubation at $25^{\circ} \mathrm{C}$ for $10 \mathrm{~min}$. After preparing the pellet fraction and the supernatant fraction, the cyt $\mathrm{c}$ content of both fractions was determined by western blotting. The percent release of cyt c (\%) was calculated from the intensities of the immunoblotted protein band of the supernatant fraction divided by the total of the supernatant and pellet fractions, multiplied by 100 . A, with phosphate; B, without phosphate.

Figure 3. Efficient cytosolic release of liposomally encapsulated Rho from Tf-L equipped with Chol-GALA. Liposomes encapsulation Rho as an aqueous phase marker were incubated with $\mathrm{K} 562$ cells at $37^{\circ} \mathrm{C}$ for $6 \mathrm{hr}$ and then analyzed by confocal laser scanning microscopy. Non-modified liposomes (A), Tf-L (B), and Tf-L equipped with Chol-GALA (C). 
Figure 4. Cytotoxic effects of MP encapsulated in liposomes. MP was added to K562 cells, followed by incubation at $37^{\circ} \mathrm{C}$ for $10 \mathrm{~min}$. Mitochondrial fraction (Mt), cytosol fraction $(\mathrm{Cy})$, and medium fraction $(\mathrm{Me})$ were obtained as described under "Materials and Methods". Each sample was analyzed by western blotting to detect cyt c. Lane 1, rat liver mitochondria (RLM); lanes 2-4, mitochondrial isolated buffer (MIB); lanes 5-7, MP (final concentration of $100 \mu \mathrm{M}$ ); lanes 8-10, Tf-L equipped with Chol-GALA encapsulating MP (final concentration of $100 \mu \mathrm{M}$ MP). Please note that the Mt fraction was six times more concentrated than the Cy fraction.

Figure 5. Intracellular trafficking of MP in Tf-L equipped with Chol-GALA. Tf-L equipped with GALA can be internalized by tumor cells via receptor-mediated endocytosis (1st step). In the endosomes, GALA can enhance the fusion between liposomes and ensosomes at a lower $\mathrm{pH}$ and MP is released to the cytosol (2nd step). MP that escapes from the endosomes can attack the mitochondria, PT is induced, and cyt $\mathrm{c}$ is released from the mitochondria (3rd step). 
Fig. 1

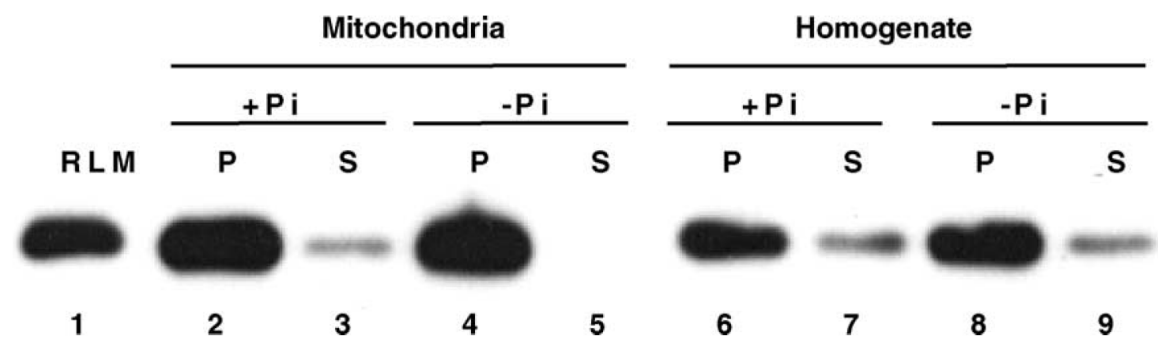


Fig. 2
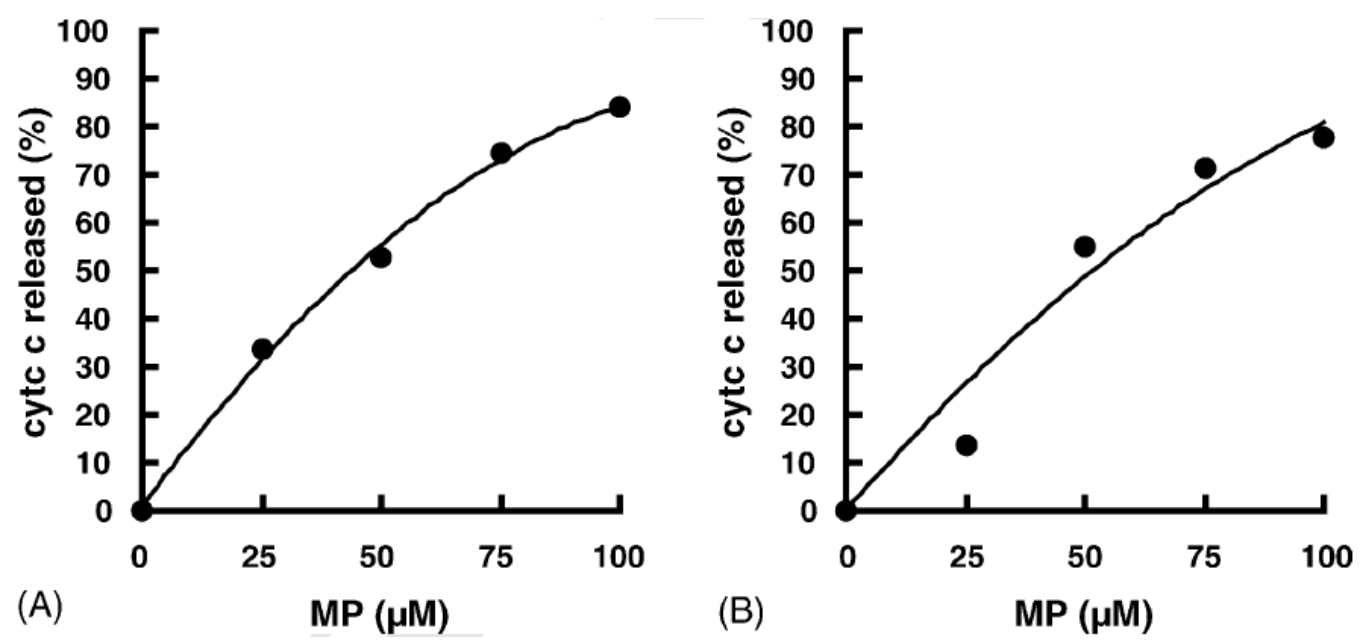
Fig. 3
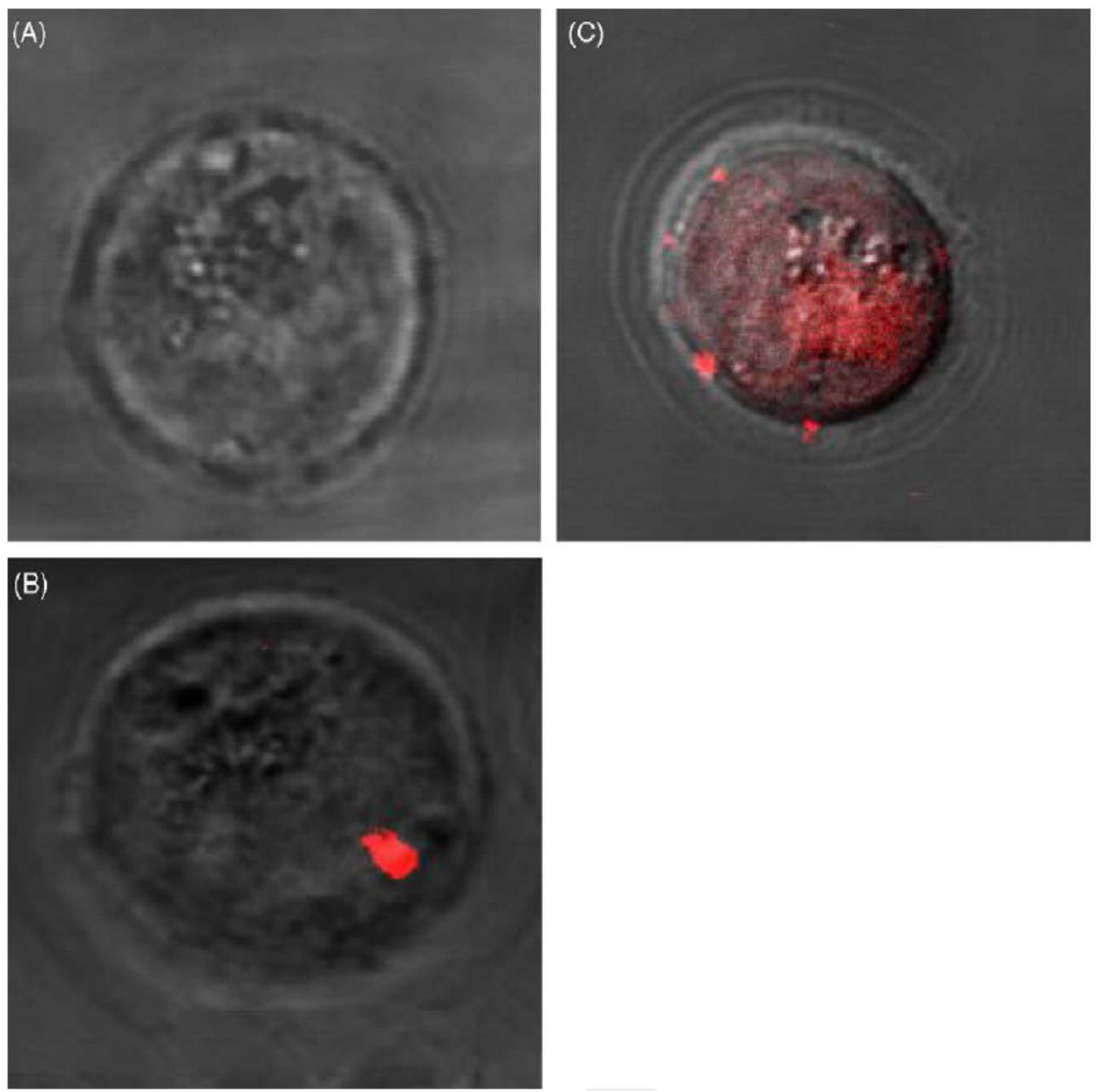
Fig. 4

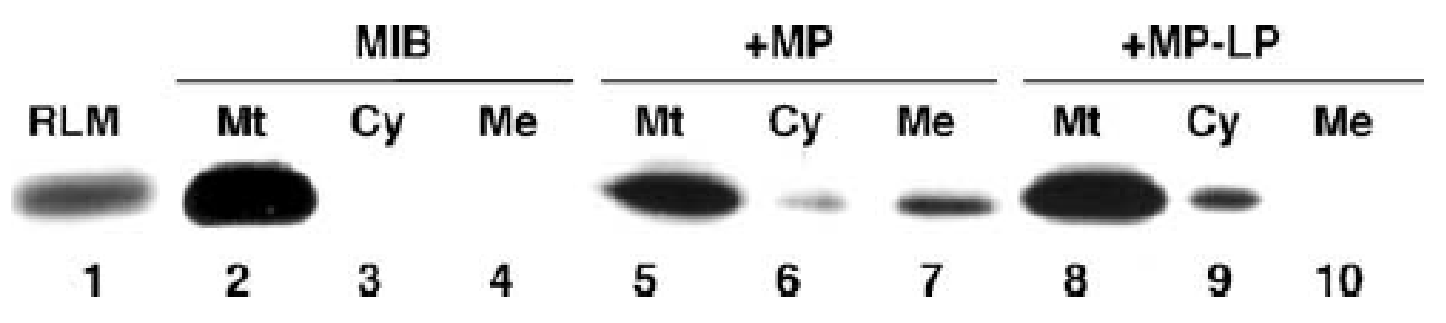


Fig. 5

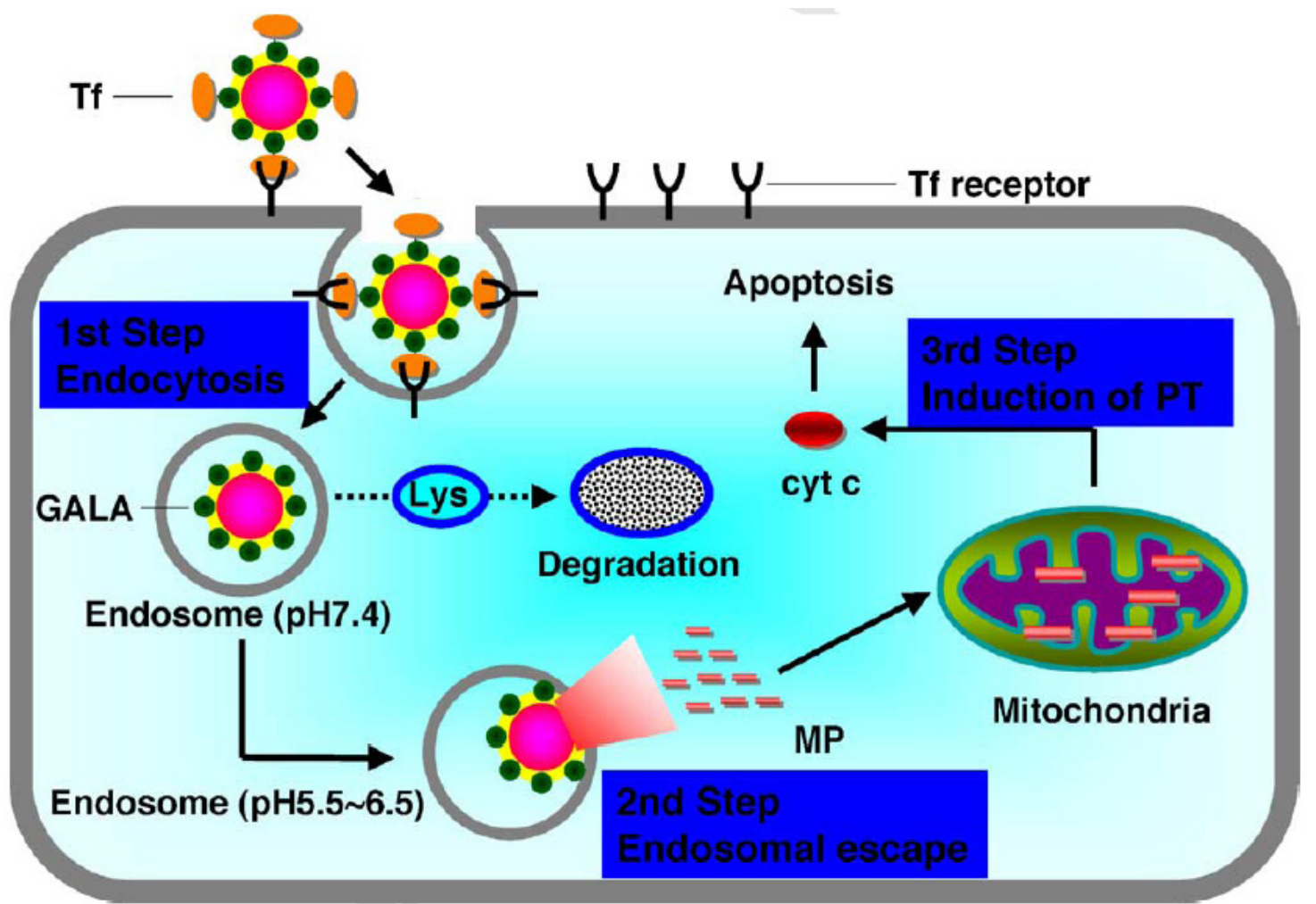

